\title{
(In)Segurança Alimentar e Nutricional em domicílios de cafeicultores de Ouro Fino, Sul de Minas Gerais, Brasil
}

\section{Bruna Carolina Silva de Souza ${ }^{1}$, Natália Pereira Silva ${ }^{2}$, Verônica Gronau Luz ${ }^{3}$, Joverlany Pessoa de Albuquerque ${ }^{4}$ e Julicristie Machado de Oliveira ${ }^{5}$}

Tendo em vista a escassez de estudos que avaliam a (In)Segurança Alimentar e Nutricional (IAN/SAN) em domicílios de trabalhadores rurais no Brasil, o objetivo deste estudo foi conhecer a prevalência de IAN/SAN entre cafeicultores de Ouro Fino, Sul de Minas Gerais. Trata-se de um estudo transversal, no qual foram realizadas entrevistas com aplicação de questionário socioeconômico e a Escala Brasileira de Insegurança Alimentar (EBIA). Observou-se uma prevalência de 51\% de IAN nos domićlíos dos cafeicultores ( $n=100)$. Não houve diferença nas prevalências de IAN segundo sexo da pessoa de referência do domicílio. As médias de renda também não foram diferentes entre os classificados ou não em IAN. Em média, os cafeicultores apresentavam

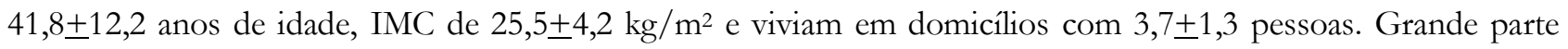
era homens $(65 \%)$, donos de seu domicílio $(95 \%)$, cultivavam exclusivamente café $(60 \%)$, trabalhavam somente em sua própria terra $(73 \%)$, referiram ter horta $(71,7 \%)$, utilizá-la para subsistência ( $96 \%)$, declararam que "arroz e feijão" eram os alimentos que não poderiam faltar (79\%) e todos faziam uso de agrotóxicos (100\%). Tais características são condizentes a uma situação de importante risco social e à saúde.

Palavras-chave: Segurança Alimentar e Nutricional, saúde da população rural, Brasil.

\footnotetext{
${ }^{1}$ Nutricionista, graduada pela Faculdade de Ciências Aplicadas - FCA/Unicamp. Endereço para correspondência: Rua Pedro Zaccaria, no 1.300, Jardim Santa Luiza, Limeira, SP. CEP: 13484-350. Tel. (19) 3701-6720._E-mail: brunacarol_51@hotmail.com

${ }^{2}$ Nutricionista, graduada pela Faculdade de Ciências Aplicadas - FCA/Unicamp. Endereço para correspondência: Rua Pedro Zaccaria, no 1.300, Jardim Santa Luiza, Limeira, SP. CEP: 13484-350. Tel. (19) 3701-6720. E-mail: natalia.pereira.s014@gmail.com

${ }^{3}$ Professora Doutora. Faculdade de Ciências da Saúde - FCS/UFGD. Endereço para correspondência: Rodovia Dourados/Itahum, Km 12 - Unidade II, Caixa Postal: 364, CEP: 79.804-970. E-mail: veronicagronauluz@gmail.com

${ }^{4}$ Nutricionista, mestranda do Mestrado Interdisciplinar em Ciências Humanas e Sociais Aplicadas da Faculdade de Ciências Aplicadas - FCA/Unicamp. Endereço para correspondência: Rua Pedro Zaccaria, no 1.300, Jardim Santa Luiza, Limeira, SP. CEP: 13484-350. Tel. (19) 3701-6720.E-mail: joverlany@gmail.com

${ }_{5}^{5}$ Professora Doutora. Faculdade de Ciências Aplicadas - FCA/UNICAMP. Endereço para correspondência: Rua Pedro Zaccaria, no 1.300, Jardim Santa Luiza, Limeira, SP. CEP: 13484-350.Tel. (19) 3701-6720.E-mail: julicristie.oliveira@fca.unicamp.br
} 


\section{Food and Nutrition (In)Security in households of coffee farmers in Ouro Fino City, South of Minas Gerais, Brazil}

Considering the lack of studies that assess the Food and Nutrition (In)Security (FNI/FNS) among rural workers, the aim of this study was to evaluate the prevalence of FNI/FNS in coffee producers from Ouro Fino, South Minas Gerais. This cross-sectional study was developed with interviews based on a socioeconomic questionnaire and the Brazilian Food Insecurity Scale (BFIS). It was observed a prevalence of $51 \%$ of FNI in the households of coffee farmers $(n=100)$. There was no difference in the prevalence of IAN according to the sex of the reference person of the household. The income means were also not different between the classified or not in IAN. On average, coffee farmers were $41.8 \pm 12.2$ years of age, with BMI of $25.5 \pm 4.2 \mathrm{~kg} / \mathrm{m}^{2}$, and lived in households with $3.7 \pm 1.3$ persons. Most of them were men $(65 \%)$, owners of their home (95\%), produced exclusively coffee $(60 \%)$, worked only in their own land $(73 \%)$, declared have a vegetable garden $(71.7 \%)$, used it for subsistence $(96 \%)$, affirmed that "rice and beans" were the foods that could not be lacking (79\%), and all used pesticides $(100 \%)$. Such characteristics are consistent with a situation of significant social and health risk.

Keywords: Food and Nutrition Security, rural health, Brazil.

\section{INTRODUÇÃO E OBJETIVOS}

No cenário brasileiro, a produção cafeeira adquiriu importância socioeconômica a partir da entrada de mudas trazidas da Guiana Francesa no século XVIII. Como o café adaptou-se com facilidade às condições climáticas e de solo, sua produção ganhou viabilidade econômica, tornando-se um dos mais importantes itens de exportação no período imperial. Inicialmente, a produção restringia-se aos estados do Pará e do Maranhão, porém houve uma significativa expansão, e outros estados, como Minas Gerais, São Paulo e Paraná, ganharam destaque ${ }^{[1]}$.

Pesquisas no campo da Saúde Coletiva e das Ciências Sociais têm apontado os impactos relevantes da carga horária de trabalho extenuante, renda insuficiente e baixa escolaridade nas condições de saúde e vida dos trabalhadores rurais[-4]. Esses trabalhadores, dependendo da atividade rural que desempenham, podem estar expostos também a desgastes físicos e nutricionais decorrentes da combinação de atividades laborais intensas com alimentação inadequada[3,4]. Outra questão relevante é a exposição aos agrotóxicos e as intoxicações que estão associadas a uma série de impactos à saúde do trabalhador rural[5].
Desde 2004, a Escala Brasileira de Insegurança Alimentar (EBIA) vem sendo utilizada nas Pesquisas Nacionais por Amostras por Domicilios (PNADs 2004, 2009, 2013) do Instituto Brasileiro de Geografia e Estatística (IBGE) para estimar a prevalência de (In)Segurança Alimentar e Nutricional (IAN/SAN) no país[ ${ }^{[}$. Esse instrumento, que é uma tradução e adaptação da escala estadunidense, passou por processo de validação para aplicação no Brasill17,8] e permite identificar diferentes níveis de gravidade da IAN: leve, moderada e grave ${ }^{[8]}$.

De acordo com os resultados dessas PNADs, entre 2004 e 2009, houve redução das proporções, de $43,7 \%$ para $35,2 \%$, de domicílios das áreas rurais em situação de IAN. Esta tendência de redução, porém, não se manteve entre os anos de 2009 e 2013, considerando que na última pesquisa a proporção observada foi de $35,3 \%\left[{ }^{[6]}\right.$.

Ainda em relação à situação de IAN em áreas rurais do Brasil, quando desdobrada em IAN leve, moderada e grave, é possível verificar algumas alterações importantes e sutis. Os resultados das PNADs apontam que a IAN leve atingia $20,2 \%$ dos domicílios em 2004, reduzindo-se para 19,5\% em 2009, e elevando-se para 21,4\% em 2013. Para a IAN 
moderada, observa-se uma redução de 14,9\% para $8,5 \%$, entre a primeira e a segunda, com estagnação entre essa e a terceira, quando se observou proporção de $8,4 \%$. Em contrapartida, a IAN grave manteve-se em redução nos anos analisados, partindo de 9,6\% em 2004, para 7,1\% em 2009, e 5,5\% em 2013[6]. Assim, houve uma migração interna às categorias de IAN, sem alteração global significativa.

Apesar desses importantes resultados divulgados por meio das PNADs, estudos mais específicos que avaliam situações de IAN/SAN particularmente em domicílios de populações rurais ainda são escassos no Brasil.

Em uma pesquisa multicêntrica realizada com esse grupo populacional no estado do Amazonas, observou-se proporções de $22,9 \% ; 17,6 \%$ e $28,2 \%$ de IAN leve, moderada e grave, respectivamente[?]. Outros pesquisadores buscaram, por meio de estudo qualitativo, conhecer qual era o entendimento atribuído aos conceitos utilizados EBIA por moradores das áreas rurais do estado de São Paulo. Os autores observaram uma compreensão abrangente do tema, com o seu reconhecimento como Direito Humano, incluindo questões como renda, trabalho, moradia, educação e saúde[10].

Tendo em vista a escassez de estudos que avaliam a IAN em domicílios de trabalhadores rurais no Brasil, bem como sua relação com as condições sociodemográficas, de trabalho e de saúde, a realização desta pesquisa tem sua pertinência justificada. Os resultados podem contribuir com o campo de Estudos Rurais que, segundo Vanderley[11], vem se pautando também pelas questões ambientais emergentes, de soberania alimentar, além do avanço em reconhecer os trabalhadores rurais como "sujeitos de direitos".

Assim, a discussão da realização da SAN como uma tradução do Direito Humano à Alimentação Adequada (DHAA), pode ganhar impulso nesse campo de estudo. Ademais, há a possibilidade da utilização desses resultados avaliação e formulação de políticas e programas públicos direcionados para a promoção da saúde e qualidade de vida dessa população. Assim, o objetivo deste estudo foi conhecer a prevalência de IAN (e SAN) em domicílios de cafeicultores do município de Ouro Fino, Sul do estado de Minas Gerais, e investigar os fatores associados.

\section{MATERIAL E MÉTODOS}

Este estudo transversal contou com o apoio do Sindicato dos Trabalhadores Rurais da cidade de Ouro Fino, Sul do estado de Minas Gerais, Brasil, que tem como atividade agrícola predominante o cultivo de café para exportação. Segundo o Censo de 2010, a população de Ouro Fino era de 31.568 habitantes, dessas 26.994 eram alfabetizadas, e o IDH foi de 0,722. Em relação à área rural, o Censo Agropecuário de 2006 identificou 142 estabelecimentos Agropecuários ${ }^{[2]}$.

O trabalho de campo foi realizado entre o período de outubro a dezembro de 2013, aos domingos e feriados. A amostra foi selecionada por conveniência. Foram convidados a participar da pesquisa 132 cafeicultores. Nas entrevistas, utilizou-se a EBIA[8], um questionário sociodemográfico, além de questões relacionadas à alimentação e nutrição (frequência de consumo de grupos de alimentos), ao perfil demográfico, social e condições de trabalho.

A EBIA é uma escala psicométrica composta por 14 questões que podem ser respondidas com sim ou não e identifica desde situações de acesso, de receio/medo de vivenciar a falta de comida/alimentos até a quando há redução da ingestão ou rompimento do padrão alimentar de forma importante. Assim, permite classificar os domicílios em quatro categorias:

SAN: “A família/domicílio tem acesso regular e permanente a alimentos de qualidade, em quantidade suficiente, sem comprometer o acesso a outras necessidades essenciais";

IAN leve: "Preocupação ou incerteza quanto acesso aos alimentos no futuro; qualidade inadequada dos alimentos resultante de estratégias que visam não comprometer a quantidade de alimentos";

IAN moderada: "Redução quantitativa de alimentos entre os adultos e/ou ruptura nos padrões de alimentação resultante da falta de alimentos entre os adultos";

IAN grave: "Redução quantitativa de alimentos entre as crianças e/ou ruptura nos padrões de alimentação resultante da falta de alimentos entre as crianças; fome 
(quando alguém fica o dia inteiro sem comer por falta de dinheiro para comprar alimentos)"[6].

Além da aplicação de questionários, foi realizada a antropometria de cada cafeicultor entrevistado, com aferição das circunferências da cintura $(\mathrm{cm})$, do quadril $(\mathrm{cm})$, do pescoço $(\mathrm{cm})$, o peso $(\mathrm{kg})$ e a estatura $(\mathrm{m})$. Todas as medidas foram realizadas em duplicata por um único pesquisador devidamente treinado.

Para aferição do peso, da estatura e das circunferências, utilizou-se respectivamente, uma balança digital portátil e fitas métricas inelásticas. Em relação à estatura, a fita foi devidamente fixada em parede lisa e sem rodapé, os cafeicultores foram medidos descalços, em posição ereta, com cinco pontos anatômicos encostados na parede e a cabeça em plano de Frankfurt[13]. A circunferência da cintura foi medida no ponto médio da última costela flutuante e a crista ilíaca.

Posteriormente, a relação cintura quadril (circunferência da cintura/circunferência do quadril) e Índice de Massa Corporal (peso/estatura ${ }^{2}$ ) foram calculados. Os pontos de corte considerados para o IMC foi: $<18,5 \mathrm{Kg} / \mathrm{m}^{2}$ baixo peso; 18,5 a 24,99 $\mathrm{Kg} / \mathrm{m}^{2}$ eutrofia; 25,0 a 29,99 $\mathrm{Kg} / \mathrm{m}^{2}$ sobrepeso; >30,0 $\mathrm{Kg} / \mathrm{m}^{2}$ obesidade ${ }^{[14]}$.

Os cafeicultores manifestaram voluntariamente o interesse em participar da pesquisa por meio de consentimento livre e esclarecido concedido com a assinatura do Termo de Consentimento Livre e Esclarecido (TCLE). Este estudo foi aprovado pelo Comitê de Ética em Pesquisa da Faculdade de Ciências Médicas (FCM) da Universidade Estadual de Campinas (Unicamp), com número de registro 70103.

Para o armazenamento e análise estatística dos dados, utilizaram-se os softwares Epi Data e Stata 9. Os dados foram inseridos em banco de dados por dupla digitação e, posteriormente, foram checados, analisados em relação à consistência.

Foi realizada análise descritiva dos dados, com cálculo de frequência e médias. Para testar a associação entre variáveis categóricas, utilizou-se o teste do Qui-quadrado e para varáveis quantitativas contínuas, utilizou-se o Coeficiente de Correlação de
Pearson e o teste $T$ de Student. Os resultados foram considerados estatisticamente significantes ao nível de $p<0,05$.

\section{RESULTADOS}

Tabela 1. Características sociodemográficas dos cafeicultores de Ouro Fino, Sul de Minas Gerais, Brasil, 2013

\begin{tabular}{|c|c|c|}
\hline Variável & $\mathbf{n}$ & $\%$ \\
\hline \multicolumn{3}{|l|}{ Idade } \\
\hline $18-24$ & 12 & 12,0 \\
\hline $25-34$ & 17 & 17,0 \\
\hline $35-44$ & 22 & 22,0 \\
\hline $45-60$ & 49 & 49,0 \\
\hline \multicolumn{3}{|l|}{ Sexo } \\
\hline Masculino & 65 & 65,0 \\
\hline Feminino & 35 & 35,0 \\
\hline \multicolumn{3}{|l|}{ Raça/cor } \\
\hline Branca & 85 & 85,0 \\
\hline Preta/Parda & 15 & 15,0 \\
\hline \multicolumn{3}{|l|}{$\begin{array}{l}\text { Escolaridade do chefe da família em } \\
\text { anos de estudo }\end{array}$} \\
\hline $2-4$ & 52 & 52,0 \\
\hline $5-8$ & 21 & 21,0 \\
\hline $9-12$ & 24 & 24,0 \\
\hline $13-17$ & 3 & 3,0 \\
\hline \multicolumn{3}{|l|}{ Bolsa Família } \\
\hline Beneficiário & 7 & 7,0 \\
\hline Não beneficiário & 93 & 93,0 \\
\hline \multicolumn{3}{|l|}{ PRONAF } \\
\hline Beneficiário & 20 & 20,0 \\
\hline Não beneficiário & 80 & 80,0 \\
\hline \multicolumn{3}{|l|}{ Trabalha com Carteira Assinada } \\
\hline Sim & 9 & 9,0 \\
\hline Não & 91 & 91,0 \\
\hline \multicolumn{3}{|l|}{ Renda per capita mensal em reais (R\$) } \\
\hline $100-399$ & 57 & 57,0 \\
\hline $400-799$ & 24 & 24,0 \\
\hline $800-1199$ & 16 & 16,0 \\
\hline $1200-1599$ & 2 & 2,0 \\
\hline 1600-1999 & 0 & 0,0 \\
\hline $2000-2500$ & 1 & 1,0 \\
\hline \multicolumn{3}{|l|}{ Relação com a propriedade rural } \\
\hline Proprietário & 62 & 62,0 \\
\hline Parceiro & 37 & 37,0 \\
\hline Usa a terra cedida por parentes ou outros & 1 & 1,0 \\
\hline \multicolumn{3}{|l|}{ O que planta atualmente } \\
\hline Somente café & 60 & 60,0 \\
\hline $\begin{array}{l}\text { Café consorciado a outras culturas (arroz, } \\
\text { milho, feijão, fava, eucalipto ou hortaliças) }\end{array}$ & 40 & 40,0 \\
\hline
\end{tabular}


Dos 132 cafeicultores convidados a participar da pesquisa, 100 consentiram mediante assinatura do TCLE. Como descrito na Tabela 1, 70\% dos entrevistados apresentavam idade entre 35-60 anos, com média de 41,8+12,2.

A maior parte dos cafeicultores (65\%) era homens, com ensino fundamental incompleto (2-4 anos de estudo) (52\%), com faixa de renda per capita mensal predominante entre 100 a 399 reais (57\%), proprietários da sua própria terra $(62 \%)$, cultivava apenas café $(60 \%)$, sem carteira assinada (91\%) e todos os cafeicultores referiram usar agrotóxicos em suas lavouras. Em relação à cobertura pelas políticas sociais e de crédito agrícola, poucos são beneficiários do Bolsa Família $(7,0 \%)$ ou do Programa Nacional de Fortalecimento da Agricultura Familiar -PRONAF $(20 \%)$.

Por meio da aplicação da EBIA, foi possível observar que $49 \%$ dos moradores dos domicílios onde viviam os cafeicultores entrevistados estavam em SAN e $51 \%$ em IAN (46\% leve e $5 \%$ grave). Após a estratificação segundo sexo da pessoa de referência do domicílio, não foram observadas diferenças nas prevalências de SAN e IAN. Não foi observada diferença significativa na renda mensal per capita média entre os cafeicultores em SAN e em IAN $(\mathrm{R} \$ 489,8+56,86$ versus $445,4+42,6 ; p=0,523) . \mathrm{Em}$ relação ao IMC, observou-se correlação positiva e estatisticamente significativa com a renda mensal per capita em reais (Gráfico 1).

Gráfico 1. Dispersão e correlação ente o IMC e a renda mensal per capita entre cafeicultores de Ouro Fino, Sul de Minas Gerais, Brasil, 2013

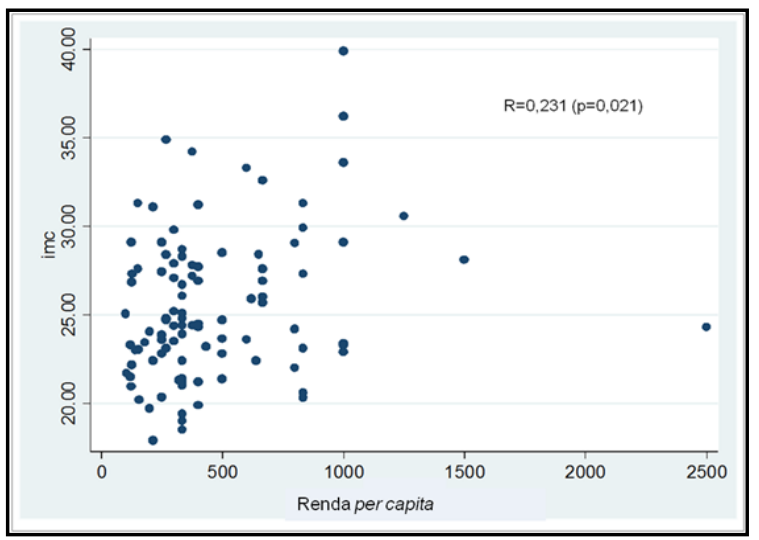

Após estratificação, observou-se que, entre os cafeicultores classificados em SAN, essa correlação não se manteve significativa $(r=0,085 ; p=0,561)$, porém a associação entre essas variáveis tornou-se mais evidente no grupo classificado em IAN $(r=0,412$; $p=0,003)$.

Assim como para o IMC, observou-se correlação estatisticamente significante entre a renda mensal per capita com a relação cintura quadril $(\mathrm{R}=0,223, p=0,026)$ e a circunferência do pescoço $(\mathrm{R}=0,319, p=0,001)$.

A associação entre a frequência de ingestão de alguns itens alimentares descritos na tabela 2 e a classificação em IAN (sim ou não) foi testada por meio do teste de Qui-quadrado, porém não se observou diferenças significativas.

Quanto ao acesso a alimentos e ao perfil de ingestão alimentar (Tabela 2), 72\% dos cafeicultores possuem horta em seu domicilio, sendo essa destinada predominantemente para alimentação familiar.

A base da alimentação dos trabalhadores é arroz e feijão, sendo descritos por eles como imprescindíveis diariamente, pois muitos relataram que “arroz e feijão não podem faltar".

A ingestão de carne vermelha é alta, cerca de $80 \%$ dos trabalhadores ingerem de 3 a 7 dias por semana. Já a de frango é bem menor, cerca de $90 \%$ da população consomem frango de 0 a 2 dias por semana.

A participação de legumes e verduras na dieta, por sua vez, encontra-se mais acentuada, em torno de $80 \%$, na faixa de frequência de 0 a 4 dias por semana. Para refrigerantes e sucos artificiais, a ingestão mostrou-se elevada, pois cerca de $55 \%$ dos cafeicultores referiram ingerir essas bebidas de 3 a 7 dias na semana. A ingestão diária de leite também foi alta $(83 \%)$. 
Tabela 2. Acesso a alimentos e frequência na ingestão alimentar em cafeicultores de Ouro Fino, Sul de Minas Gerais, Brasil, 2013

\begin{tabular}{|c|c|c|}
\hline Variável & $\mathbf{n}$ & $\%$ \\
\hline \multicolumn{3}{|l|}{ Possui horta em casa } \\
\hline Sim & 72 & 72,0 \\
\hline Não & 28 & 28,0 \\
\hline \multicolumn{3}{|l|}{ Destino do que se planta na horta } \\
\hline Alimentação familiar & 67 & 93,2 \\
\hline Vende & 3 & 4,1 \\
\hline Ambos & 2 & 2,7 \\
\hline \multicolumn{3}{|l|}{$\begin{array}{l}\text { No mês anterior, não comprou algum alimento } \\
\text { devido o preço }\end{array}$} \\
\hline Sim & 23 & 23,0 \\
\hline Não & 77 & 77,0 \\
\hline \multicolumn{3}{|l|}{ Alimentos que não podem faltar em casa } \\
\hline Arroz e feijão & 79 & 79,0 \\
\hline $\begin{array}{l}\text { Arroz, feijão e outros (carne, macarrão, óleo, salada, } \\
\text { farinha, café, leite, queijo e tempero) }\end{array}$ & 18 & 18,0 \\
\hline Feijão ou leite ou fruta e hortaliça & 3 & 3,0 \\
\hline \multicolumn{3}{|l|}{ Consumo de feijão } \\
\hline $0-2$ dias & 1 & 1,0 \\
\hline 3-4 dias & 1 & 1,0 \\
\hline $5-7$ dias & 98 & 98,0 \\
\hline \multicolumn{3}{|l|}{ Ingestão de verdura ou legume } \\
\hline $0-2$ dias & 51 & 51,0 \\
\hline 3-4 dias & 28 & 28,0 \\
\hline 5-7 dias & 21 & 21,0 \\
\hline \multicolumn{3}{|l|}{$\begin{array}{l}\text { Ingestão de salada (alface e tomate ou outras } \\
\text { hortaliças cruas) }\end{array}$} \\
\hline $0-2$ dias & 36 & 36,0 \\
\hline 3-4 dias & 46 & 46,0 \\
\hline 5-7 dias & 18 & 18,0 \\
\hline \multicolumn{3}{|l|}{ Ingestão de verdura ou legume cozido } \\
\hline $0-2$ dias & 58 & 58,0 \\
\hline 3-4 dias & 30 & 30,0 \\
\hline 5-7 dias & 12 & 12,0 \\
\hline \multicolumn{3}{|l|}{ Ingestão de carne vermelha } \\
\hline $0-2$ dias & 19 & 19,0 \\
\hline 3-4 dias & 42 & 42,0 \\
\hline $5-7$ dias & 39 & 39,0 \\
\hline \multicolumn{3}{|l|}{ Ingestão de frango ou galinha } \\
\hline $0-2$ dias & 91 & 91,0 \\
\hline 3-4 dias & 8 & 8,0 \\
\hline 5-7 dias & 1 & 1,0 \\
\hline \multicolumn{3}{|l|}{ Ingestão de suco de fruta } \\
\hline $0-2$ dias & 69 & 69,0 \\
\hline 3-4 dias & 10 & 10,0 \\
\hline 5-7 dias & 21 & 21,0 \\
\hline \multicolumn{3}{|l|}{ Ingestão de fruta } \\
\hline $0-2$ dias & 36 & 36,0 \\
\hline 3-4 dias & 33 & 33,0 \\
\hline 5-7 dias & 31 & 31,0 \\
\hline \multicolumn{3}{|l|}{ Ingestão de refrigerante ou suco artificial } \\
\hline $0-2$ dias & 43 & 43,0 \\
\hline 3-4 dias & 21 & 21,0 \\
\hline 5-7 dias & 36 & 36,0 \\
\hline \multicolumn{3}{|l|}{ Ingestão de leite } \\
\hline $0-2$ dias & 16 & 16,0 \\
\hline 3-4 dias & 1 & 1,0 \\
\hline 5-7 dias & 83 & 83,0 \\
\hline \multicolumn{3}{|l|}{ Ingestão de bebida alcoólica } \\
\hline $0-2$ dias & 93 & 93,0 \\
\hline 3-4 dias & 4 & 4,0 \\
\hline 5-7 dias & 3 & 3,0 \\
\hline
\end{tabular}

\section{DISCUSSÃO}

O presente estudo investigou a prevalência de IAN/SAN em domicílios de cafeicultores do município de Ouro Fino, Sul de Minas Gerais, por meio da aplicação de uma escala validada para o Brasil, a EBIA. Observaram-se prevalências de $51 \%$ de IAN (46\% leve e 5\% grave) e de $49 \%$ de SAN. Assim, as prevalências de IAN nessa população foram maiores do que as encontradas na última PNAD de 2013 para a população rural brasileira: 35,3\% (21,4\% leve, 8,4\% moderada e $5,5 \%$ grave $)^{[6]}$.

De acordo com estimativa da Organização das Nações Unidas para Alimentação e Agricultura (FAO), cerca de $70 \%$ populações em IAN no mundo vivem em áreas rurais ${ }^{[15]}$. As altas prevalências de IAN em áreas rurais não devem ser justificadas somente pela escassez ou dificuldades de acesso a alimentos, mas também pela falta de investimento e de condições que garantam a SAN, por meio de programas de transferência de renda ou incentivo à produção para autoconsumo [16,17].

Dentre os cafeicultores entrevistados no presente estudo, $57 \%$ declararam pertencer a faixa de renda per capita mensal que variava entre 100 a 399 reais. Apesar de não ter sido observada diferença significativa na renda mensal per capita média entre os cafeicultores em SAN e em IAN, outros estudos apontam que a renda é um dos fatores que melhor indicam o grau de severidade da IAN. Um estudo realizado em Duque de Caxias, Rio de Janeiro, no qual $53,8 \%$ das familias foram classificadas em algum grau de IAN, apontam que o aumento da renda per capita esteve associado à redução na proporção de IAN. Segundo os autores, $23,1 \%$ dos domicílios com renda per capita de um salário mínimo ou mais foram classificados em IAN, sendo $18,1 \%$ leve e $5 \%$ moderada ${ }^{[18]}$.

Outro estudo, realizado em unidades básicas de saúde das regiões Sul e Nordeste do Brasil, também observou maior probabilidade IAN moderada e grave em domicílios com menor renda familiar per capita e entre os beneficiários de programas de transferência de renda, como o Bolsa Família. Os autores relataram que uma renda per capita mensal de no mínimo $\mathrm{R} \$ 175$ reduziria em $45,4 \%$ a IAN grave ou moderada no Sul e em $59,5 \%$ no Nordeste ${ }^{[19]}$.

Em relação ao acesso às políticas públicas, observou-se que apenas 7\% dos domicílios dos cafeicultores entrevistados eram beneficiários do Bolsa Familia e $20 \%$ do PRONAF. Provavelmente, a prevalência de IAN encontrada no presente estudo 
poderia estar reduzida se houvesse maior abrangência e efetividade dessas políticas públicas, especialmente se os programas pudessem ser moldados de acordo com as características da região em que estão implementados, com uma análise mais sensível da importância e do impacto dos benefícios no local onde está inserido[20].

Foi observada uma correlação positiva e estatisticamente significativa entre renda per capita em reais e IMC apenas no grupo de cafeicultores classificados em IAN. O duplo fardo, ou seja, tanto prevalências importantes de baixo peso quanto de obesidade podem estar associadas à IAN. Muitos fatores podem condicionar esse complexo quadro, como por exemplo o aumento da disponibilidade de consumo de alimentos industrializados e de reduzida qualidade nutricional para populações de baixa renda e com condições de vida desfavoráveis[21].

Em estudo realizado em quatro assentamentos do estado de Sergipe, observou-se, além de alta prevalência de IAN (88,8\%; sendo 15,1\% grave), associação com baixa renda per capita e alimentação com variedade reduzida ${ }^{[2]}$. No entanto, entre os cafeicultores entrevistados, a variedade de alimentos consumidos não se associou à IAN. Apesar dessa ausência de associação, observou-se alto consumo de carne de vaca, suco artificial e refrigerante. Porém, destaca-se a permanência de características da dieta tradicional brasileira, com alta prevalência de consumo diário de "arroz com feijão" e presença de horta na maior parte dos domicílios rurais investigados, o que pode potencializar a SAN.

\section{CONCLUSÃO}

Por meio deste estudo, foi possível identificar alta prevalência de IAN (51\%) em domicílios de cafeicultores do município de Ouro Fino, Sul de Minas Gerais. Apesar de a renda não ter sido identificada como um condicionante, outros estudos realizados com diferentes grupos populacionais a apontam como fator associado à IAN. Nesse quadro de alta prevalência, destaca-se que poucos cafeicultores são beneficiários de políticas públicas de SAN, o que indica falhas na identificação de populações que atenderiam os critérios de inclusão. Tais características são condizentes a uma situação de importante risco social e à saúde. Apesar de não ter sido observada uma relação entre IAN/SAN e variedade de alimentos, verificou-se uma convivência de alto consumo de sucos artificiais e refrigerantes com a manutenção de características da dieta tradicional, como o "arroz com feijão" e o hábito de manter uma horta.

\section{REFERÊNCIAS}

[1] Brasil. Ministério da Agricultura, Pecuária e Abastecimento Café [Internet]. 2016 [Acesso em 4 set 2018). Disponível em: http://www.agricultura.gov.br/agroestatisticas/cafe

[2] Novaes RB. Forasteiros: Trajetórias, experiências de trabalho e práticas de deslocamento dos trabalhadores do café no Alto Paranaîba (MG) [dissertação]. Rio de Janeiro: Universidade Federal Rural do Rio de Janeiro; 2009.

[3] Luz VG, Corrêa-Filho HR, Silva AJN, Laat EF, Vilela RAG, Silva FOC, Lia TOZ. Migrant labor and wear-out in manual sugarcane harvesting in São Paulo, Brazil. Ciência \& Saúde Coletiva. 17(10):2831-2840; 2012.

[4] Luz VG, Lia TOZ, Vilela RAG, Corrêa-Filho HR. Consumo alimentar e condições de trabalho no corte manual de cana de açúcar no estado de São Paulo. Revista Saúde e Sociedade. 23(4):1316-1328; 2014.

[5] Malaspina FG, ZiniLise ML, Bueno PC. Perfil epidemiológico das intoxicações por agrotóxicos no Brasil, no período de 1995 a 2010. Caderno de Saúde Coletiva. 19(4):425-434; 2011.

[6] IBGE. Pesquisa Nacional por Amostra de Domicílios; Segurança Alimentar [Internet]. 2014. [Acesso em 15 mar 2018]. Disponível em: https://biblioteca.ibge.gov.br

[7] Pérez-Escamilla R, Segall-Corrêa AM, Maranha LK, Sampaio MFA, Marín-León L, Panigassi G. An Adapted Version of the U.S. Department of Agriculture Food Insecure Module Is a Valid Tool for assessing household food insecurity in Campinas, Brazil. Journal of Nutrition. 134(8):1923-8192; 2004.

[8] Segall-Corrêa AM, Marin-Leon L. A Segurança Alimentar no Brasil: proposição e usos da escala brasileira de medida da insegurança alimentar (EBIA) de 2003 a 2009. Segurança Alimentar e Nutricional. 16(2):1-19; 2009.

[9] Yuyama LKO, Aguiar JPL, Pantoja L, Maeda RN, Melo T, Alencar FH et al. Segurança/insegurança alimentar em famílias urbanas e rurais no estado do Amazonas: I. Validação de metodologia e de instrumento de coleta de informação. Acta Amazônica. 37(2):247-252; 2007.

[10] Sampaio MFA, Kepple AW, Segall-Corrêa AM, Oliveira JTA, Panigassi G, Maranha LK et al. (In)Segurança Alimentar: experiência de grupos focais com populações rurais do Estado de São Paulo. Segurança Alimentar e Nutricional. 13(1):64-77; 2006. 
[11] Vanderley MNB. Um saber necessário: os estudos rurais no Brasil. Campinas: Editora da Unicamp; 2011.

[12] IBGE. IBGE Cidades [Internet]. 2018 [Acesso em 4 set 2018]. Disponível em: https://cidades.ibge.gov.br

[13] Laboratório de Avaliação Nutricional de Populações LANPOP/HNT/FSP/US. Guia para realização de medidas antropométricas [Internet]. 2015 [Acesso em 13 jan 2015]. Disponível em: http://hygeia.fsp.usp.br/lanpop/manual.pdf

[14] WHO. Obesity: preventing and managing the global epidemic. Report of a WHO Consultation. WHO Technical Report Series 894. Geneva: World Health Organization; 2000.

[15] Food and Agriculture Organization of the United Nations (FAO). Statistical Yearbook. Rome: FAO; 2013.

[16] Hirai WG, Anjos FS. Estado e segurança alimentar: alcances e limitações de políticas públicas no Brasil. Revista Virtual Textos \& Contextos. 6(8):1-9; 2007.

[17] Mondini L, Rosa TE, Gubert MB, Sato GS, Benício MHD. Insegurança alimentar e fatores sociodemográficos associados nas áreas urbana e rural do Brasil. Informações Econômicas. 41(2):52$60 ; 2011$.

[18] Salles-Costa R, Pereira RA, Vasconcellos MTL, Veiga GV, Marins VMR, Jardim BC et al. Association between socioeconomic factors and food insecurity: a population-based study in the Rio de Janeiro metropolitan area, Brazil. Revista de Nutrição. 21(Suppl): 99s-109s; 2008.

[19] Facchini LA, Nunes BP, Motta JVS, Tomasi E, Silva SM, Thumé E et al. Insegurança alimentar no Nordeste e Sul do Brasil: magnitude, fatores associados e padrões de renda per capita para redução das iniquidades. Cadernos de Saúde Pública. 30(1):161-174; 2014.

[20] Fonseca C, Scalco LM, Castro HC. Etnografia de uma política pública: controle social pela mobilização popular. Horizontes Antropológicos. 24(50):271-303; 2018.

[21] Morais DC, Dutra LV, Franceschini SCC, Priore ES. Insegurança alimentar e indicadores antropométricos, dietéticos e sociais em estudos brasileiros: uma revisão sistemática. Ciência \& Saúde Coletiva. 19(5):1475-1488; 2014.

[22] Almeida JA, Santos AS, Nascimento MAO, Oliveira JVC, Silva DG, Mendes-Netto RS. Fatores associados ao risco de insegurança alimentar e nutricional em famílias de assentamentos rurais. Ciência \& Saúde Coletiva. 22(2):479-488; 2017. 